\title{
RELATION BETWEEN FILTRATION AND SOIL CONSOLIDATION THEORIES
}

\author{
TOMASZ STRZELECKI \\ Wrocław University of Technology, Institute of Geotechnics and Hydrotechnics, \\ Wybrzeże Wyspiańskiego 27, 50-370 Wrocław, Poland, e-mail: Tomasz.Strzelecki@pwr.edu.pl

\section{MICHAŁ STRZELECKI} \\ KGHM CUPRUM, Ltd. Research and Development Centre, \\ ul. Gen. Wł. Sikorskiego 2-8, 53-659 Wrocław, Poland, e-mail: mstrzelecki@cuprum.wroc.pl
}

\begin{abstract}
This paper presents a different, than commonly used, form of equations describing the filtration of a viscous compressible fluid through a porous medium in isothermal conditions. This mathematical model is compared with the liquid flow equations used in the theory of consolidation. It is shown that the current commonly used filtration model representation significantly differs from the filtration process representation in Biot's and Terzaghi's soil consolidation models, which has a bearing on the use of the methods of determining the filtration coefficient on the basis of oedometer test results. The present analysis of the filtration theory equations should help interpret effective parameters of the non-steady filtration model. Moreover, equations for the flow of a gas through a porous medium and an interpretation of the filtration model effective parameters in this case are presented.
\end{abstract}

Key words: filtration, seepage, soil consolidation, underground water

\section{INTRODUCTION}

\subsection{LAWS GOVERNING WATER SEEPAGE THROUGH PORES OF TWO-PHASE MEDIUM}

All rocks, especially soils, have permeability properties owing to which liquids and gases can penetrate through them under the influence of: the gravitational field, an electric or chemical potential difference and a temperature difference. The basic law of the flow of water through soils was formulated by H. Darcy [12] in the years 1852-1855. Darcy did not take into account earlier works (1842) by J.L. Poiseuille, who when theoretically considering the laminar flow of water through a capillary tube had derived an averaged equation of motion, similar to Darcy's equation. Darcy's law in its original form is expressed by the formula

$$
\vec{v}=k \vec{J}
$$

where $k$-a filtration coefficient, $\vec{v}-$ a vector field of seepage velocity, and $J-$ a hydraulic gradient defined by the formula

$$
\vec{J}=\operatorname{grad}(H)
$$

where $H$ stands for a hydraulic head expressed by the simplified Bernoulli formula

$$
H=\frac{p}{\rho g}+x_{i} \delta_{i 3}
$$

where $p$ - pressure, $\rho$ - bulk density and $g$ - acceleration due to gravity.

In many papers [15], [26], [27] critical comments on Darcy's law have been made, which can be summarized as follows:

- Darcy did not take into account the fact that the sense of hydraulic head gradient $H$ is opposite to that of seepage velocity $\vec{v}$ and so basic Darcy's law should have the form

$$
\vec{v}=-k \vec{J}
$$

- In 1884, G. Kröber (acc. to Wieczysty [27]) raised the issue of the limits of the applicability of Darcy's law.

- In 1901, Forchheimer [15] presented the following relation defining the filtration flow equation in the 
case when the motion of the liquid flowing through pores has a turbulent character

$$
\vec{J}=a \vec{v}+b \vec{v} \vec{v} .
$$

All the aspects and comments relating to Darcy's law can be found in the works by Polubarinov-Koczina [22], A. Wieczysty [27], T. Strzelecki et al. [26], and many others.

The problem of laminar flow through the pores of the soil medium was also analyzed by the asymptotic homogenization method consisting in mathematical upscaling of the processes taking place at the pore scale. On the heterogeneity scale the laminar flow of a liquid is described, in accordance with the works of Bensousan et al. [7], Auriault et al. [1], [3], Strzelecki et al. [25], Łydżba [19], [20], by the Navier-Stockes equations in conjunction with the equation for the continuity of the flow of an incompressible liquid for which $\operatorname{div}(\vec{v})=0$. Taking into account boundary condition $(\vec{v})=0$ at the liquid/solid interface and the periodicity conditions for the vector velocity field and the function of the pressure in the liquid one gets a system of differential equations which is the starting point for the homogenization process. The solution leads to Darcy's linear law with a mathematical description showing how to numerically determine the value of the permeability tensor if one can determine the porous medium structure and knows the viscosity of the liquid flowing through the pores of the medium. The solution obtained in the works cited above is a significant achievement of theoretical physics. Using only mathematical tools by making the passage from the microscopic scale to the macroscopic scale a completely different character of the equations describing the flow in the two scales was obtained and the order of magnitude of the permeability tensor in the macroscopic scale was determined. It should be noted that the obtained result fully agrees with the experimental results, and the dependence between the filtration coefficient and ratio $l^{2} / \mu$, where $l$ defines the size of the representative volume element (RVE) and $\mu$ stands for the viscosity of the liquid flowing through the pores of the medium, has been confirmed by numerous experiments.

\subsection{CLASSIC MATHEMATICAL MODEL OF FILTRATION THEORY}

The equations of the hydrodynamics of underground waters were determined under the following assumptions:
- a porous medium forms the structure of a solid body, considered to be a continuous medium, inside of which there exists a network of interconnected pore channels;

- closed pores, containing a liquid or a gas, do not occur;

- the network of pore channels is regular enough for determining a representative volume element (RVE) to represent an isolated cuboid with infinitely small dimensions;

- the pores of the medium are filled with water;

- the flow of the liquid takes place at a constant temperature;

- neither the Earth's electric field nor its magnetic field has an influence on the filtration process;

- the influence of the chemical potential is not taken into account;

- the motion of the liquid is analyzed with respect to fixed reference system $x_{i}$, i.e., in the Lagrangian coordinate system.

The behaviour of the liquid is described by:

- constitutive equations of state,

- equations of flow continuity,

- equations of the motion of the liquid through the porous medium.

As will be shown, the above system of equations made it possible to create a mathematical model of the flow of the liquid through the porous medium. The equations derived must be completed with boundary and initial conditions.

\subsubsection{CONSTITUTIVE EQUATIONS OF STATE}

A fluid with high volumetric compressibility (e.g., a gas or a mixture of a liquid and a gas) or a liquid characterized by very low compressibility can flow through the pores of a porous medium. Let us limit our considerations to two cases of the equation of state: (1) when there is a liquid in the pores of the two-phase medium, and (2) when the pores are filled with a gas.

In both cases the pressure prevailing in the fluid or its increase causes volume strains in both the fluid and the solid phase of the medium. The process in which volumetric changes of the skeleton are taken into account, is referred to as an elastic regime of the filtration flow. The so called rigid filtration regime is associated with the case in which the effects of the elasticity of the skeleton are not taken into account.

It is assumed that the solid phase of the medium does not undergo deviatoric deformations and only volumetric changes, manifesting themselves in a change in 
the porosity of the solid body matrix, are assumed at this stage of the analysis.

The volume elastic change of the fluid is described by a relation according to which a change in fluid bulk density $\rho$ is proportional to the change in the pressure prevailing in the fluid

$$
\frac{d \rho}{\rho}=\beta_{w} d p
$$

where $\beta_{w}$ - a coefficient of the volumetric compressibility of the fluid, defined as a relative change in the volume of the fluid by 1 bar. For example, for water $\beta_{w}$ amounts to $10^{-10} 1 / \mathrm{Pa}$.

Equation (6) leads to the following nonlinear dependence between fluid density and liquid pressure:

$$
\rho=\rho_{a} \exp \left(\beta_{w} p\right)
$$

where $\rho_{a}$ stands for the density of the fluid in atmospheric pressure conditions.

At small pressures (up to 100 bar) one can assume that the changes in density are small enough and so $\rho=$ const.

\subsubsection{EQUATION OF FLOW CONTINUITY}

Let $\Omega$ stand for an elementary domain filled with a two-phase medium. Let us denote the boundary surface through which the filtration flow of the liquid takes place with $S$. Let $\vec{n}$ stand for a versor normal to $S$ and directed outside of area $\Omega$.

The flow of the liquid through surface $S$ bounding the volume $\Omega$ (Fig. 1) is defined by the equation

$$
\int_{S} \rho v_{i} d S+\int_{\Omega} \frac{\partial(\rho)}{\partial t} d \Omega=0 .
$$

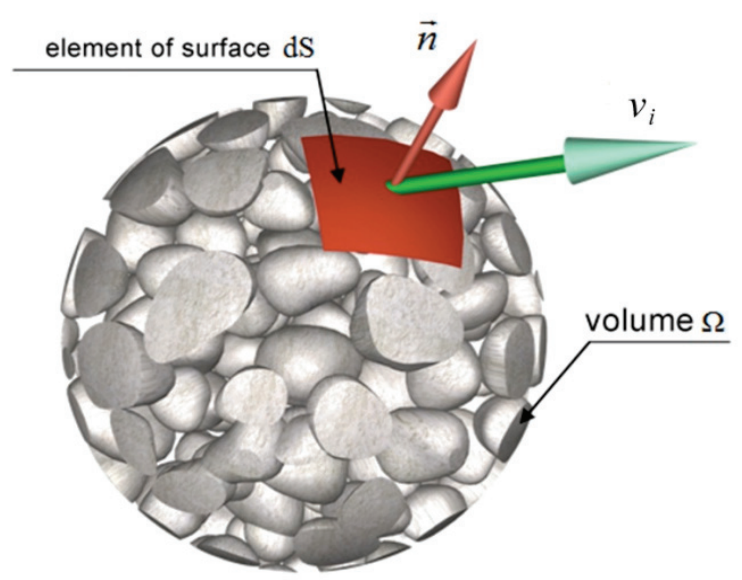

Fig. 1. Flow of medium through surface $S$ bounding volume - after Bartlewska [4]
Using the Gauss-Ostrogradsky theorem one can replace the surface integral with a volume integral. Thus one gets

$$
\int_{\Omega} \frac{\partial\left(\rho v_{i}\right)}{\partial x_{i}} d \Omega+\int_{\Omega} \frac{\partial(\rho)}{\partial t} d \Omega=0
$$

Thanks to the above equation one can write the following local relation

$$
\frac{1}{\rho} \frac{d \rho}{\rho}+\operatorname{div}(\vec{v})=0
$$

where

$$
\frac{d^{\bullet}}{d t}=\frac{\partial^{\bullet}}{\partial t}+v_{i} \frac{\partial^{\bullet}}{\partial x_{i}}
$$

defines the material derivative of the bulk density of the fluid.

\subsubsection{CLASSIC MATHEMATICAL MODEL OF FILTRATION PROCESS}

Assuming in the first case that the soil medium is an ideally rigid body and the liquid flowing through the network of filtration channels is incompressible, the system of equations describing the laminar flow comes down to the equations of state: $\rho=$ const, the flow continuity equation: $\operatorname{div}(\vec{v})=0$ and the motion equations expressed by Darcy's law: $\vec{v}=-\widetilde{k} \operatorname{grad}(H)$ where $\tilde{k}$ denotes a permeability tensor. Substituting the equations of motion into the flow continuity equation one gets the following differential equation describing the flow of an incompressible liquid through a non-deformable isotropic homogenous porous medium

$$
\operatorname{div}(\tilde{k} \operatorname{grad}(H))=0
$$

Equation (12) is the basic equation of the theory of filtration for the case when the soil medium is an ideally rigid body.

In the second case, it is assumed that the soil medium exhibits volumetric deformability as regards both its solid phase and liquid phase, but it does not exhibit deviatoric deformability. The flow continuity equation in this case is expressed by equation (10). Substituting Darcy's equation of motion into equation (10) and taking into account the fact that $\rho=\rho_{c} f$ one gets the following classic equation of the non-steady flow

$$
\operatorname{div}\left(\rho_{c} f \tilde{k} \operatorname{grad}(H)\right)=\frac{\partial\left(\rho_{c} f\right)}{\partial t}
$$

where $\rho_{c}$ - specific density of fluid, f-porosity. 
The derivative on the right side of equation (13) is the product of the two functions and so

$$
\frac{\partial\left(\rho_{c} f\right)}{\partial t}=\rho_{c} \frac{\partial f}{\partial t}+f \frac{\partial \rho_{c}}{\partial t} .
$$

Then it is assumed that the rate of the change in fluid density over time depends on the product of the density and the rate of the change in pressure

$$
\frac{\partial \rho_{c}}{\partial t}=\rho_{c} \beta_{w} \frac{\partial p}{\partial t}
$$

which can be recognized as consistent with constitutive relation (6), assuming that the change in density is a change in density over time. Assuming further that the rate of the change in porosity is directly proportional to the rate of the change in the pressure of the liquid (as in the classic filtration theory), the following constitutive relation is introduced

$$
\frac{\partial f}{\partial t}=\beta_{s} \frac{\partial p}{\partial t}
$$

It is difficult to substantiate the above relation by means of any phenomenological relation belonging to soil and rock mechanics. Biot's consolidation theory directly assumes that porosity in the soil skeleton deformation process is a constant quantity, which is not consistent with reality, but it does not significantly affect the validity of this theory's solutions. Using formulas (15) and (16) one can present relation (14) in the form

$$
\frac{\partial\left(\rho_{c} f\right)}{\partial t}=\rho_{c}^{2} g \beta_{s} \frac{\partial H}{\partial t}+f \rho_{c}^{2} g \beta_{w} \frac{\partial H}{\partial t} .
$$

In the classic mathematical model it is then assumed that

$$
\frac{\partial p}{\partial t}=f \rho_{c} g \frac{\partial H}{\partial t}
$$

which obviously is inaccurate since adopting as valid the definition of hydraulic head for the filtration flow

$$
H=\frac{p}{f \rho_{c} g}+x_{i} \delta_{i 3}
$$

the derivative over time is

$$
\frac{\partial\left(\rho_{c} f g H\right)}{\partial t}=\rho_{c} g f \frac{\partial H}{\partial t}+H g f \frac{\partial \rho_{c}}{\partial t}+H \rho_{c} g \frac{\partial f}{\partial t},
$$

hence the rate of pressure variation is expressed by the formula

$$
\frac{\partial p}{\partial t}=f \rho_{c} g \frac{\partial H}{\partial t}+g f H \frac{\partial \rho_{c}}{\partial t}+g H \rho_{c} g \frac{\partial f}{\partial t}-x_{i} g \delta_{i 3} \frac{\partial \rho_{c}}{\partial t}
$$

which leads to a following relation

$$
\frac{\partial p}{\partial t}=\frac{f \rho_{c} g \frac{\partial H)}{\partial t}}{1-\rho_{c} g \beta_{w} f H-\rho_{c} g \beta_{s} H+x_{i} \delta_{i 3} \rho_{c} g \beta_{w}} .
$$

Yet despite the significant inconsistency, the classic equation of the non-steady flow is assumed in the form

$$
\operatorname{div}(\rho \tilde{k} \operatorname{grad}(H))=\rho \eta_{s p r} \frac{\partial H}{\partial t}
$$

where $\eta_{s p r}$ is referred to as elastic capacity and amounts to

$$
\eta_{s p r}=\rho_{c} g \beta_{s}+f \rho_{c} g \beta_{w} .
$$

Assuming that the changes in the density of the liquid depending on the spatial variables were small, they were assumed to be independent of the spatial variables. Then the equation simplifies to a form

$$
\operatorname{div}(\tilde{k} \operatorname{grad}(H))=\eta_{s p r} \frac{\partial H}{\partial t}
$$

Despite the mathematical and physical inconsistencies demonstrated the above equation is regarded as the fundamental equation of the underground water hydrodynamics for a compressible medium through which a compressible liquid flows, and it is the basis for numerical computations performed using professional hydrogeological software, such as ModFlow [21].

\section{PROPOSED MATHEMATICAL MODEL OF FILTRATION THEORY}

\subsection{ASSUMPTIONS}

The assumptions for the theory of filtration, presented in the preceding section will be completed with additional definitions, stemming from Biot's consolidation theory [8], [9] and also based on Coussy's works [10], [11], for a two-phase medium. Using denotations consistent with works [25], [26] by Strzelecki et al.,

- Let $\Omega$ be a cuboidal space with infinitely small edges filled with a two-phase medium consisting 
of a porous elastic skeleton and a liquid filling its pores; let us denote the boundary of spatial element $\Omega$ with $S$ while vector $\vec{n}$ is a unit vector normal to surface $\mathrm{S}$, directed outside of element $\Omega$; $\vec{v}^{l}$ and $\vec{v}^{s}$ stand for respectively fluid seepage velocity and medium skeleton velocity and $v_{i}^{r}=v_{i}^{l}-v_{i}^{s}$ defines the components of the relative velocity of the filtration flow of the liquid through the porous medium; if $\rho_{s}$ and $\rho_{l}$ stand for the specific density of respectively the skeleton and the liquid, then the volumetric density of the skeleton $\left(\rho_{1}\right)$ and the liquid $\left(\rho_{2}\right)$, each of them related to the total volume of area $\Omega$, can be determined; denoting volumetric porosity with $f$ one can calculate the densities: $\rho_{1}=(1-\mathrm{f}) \rho_{s}$ and $\rho_{2}=f \rho_{l} ; \rho$ denotes two-phase medium density equal to the sum: $\rho=$ $\rho_{1}+\rho_{2} ; \bar{\rho}$ stands for the density of the fluid flowing through $\mathrm{S}: \bar{\rho}=f_{A} \rho_{l}$, where $f_{A}$ stands for surface porosity.

- The kinetic energy of the two-phase medium can be expressed by the formula

$$
2 K=\int_{\Omega}\left(\rho_{11} v_{i}^{s} v_{i}^{s}+2 \rho_{12} v_{i}^{s} v_{i}^{l}+\rho_{22} v_{i}^{l} v_{i}^{l}\right) d \Omega
$$

with conditions:

$$
\rho_{11}+\rho_{12}=\rho_{1}>0 ; \quad \rho_{22}+\rho_{21}=\rho_{2}>0 ; v \quad \rho_{12}<0,
$$

where $\rho_{12}$ is a new parameter with a density dimension, defining the dynamic coupling between the two phases of the medium.

- The dissipation function is a quadratic form dependent on the relative velocity of the filtration flow, which can be expressed as

$$
2 W_{d}=\int_{\Omega} b v_{i}^{r} v_{i}^{r} d \Omega
$$

where $b$ is a filtration resistance coefficient satisfying condition $b>0$.

- Using equation (261) one can determine the internal volume forces resulting from the viscous resistance of the liquid flowing through the pores of the medium; the forces acting on the skeleton of the medium are

$$
M_{i}^{s}=\frac{\partial W_{d}}{\partial v_{i}^{s}}=-\int_{\Omega} b v_{i}^{r} d \Omega
$$

and the forces acting on the liquid are

$$
M_{i}^{l}=\frac{\partial W_{d}}{\partial v_{i}^{l}}=\int_{\Omega} b v_{i}^{r} d \Omega .
$$

- The components of the local vector of skeleton momentum can be calculated from the formula

$$
\begin{aligned}
& P_{i}^{s}=\int_{\Omega}\left(\rho_{11} v_{i}^{s}+\rho_{12} v_{i}^{l}\right) d \Omega, \\
& P_{i}^{l}=\int_{\Omega}\left(\rho_{12} v_{i}^{s}+\rho_{22} v_{i}^{l}\right) d \Omega .
\end{aligned}
$$

\subsection{EQUATIONS OF FLOW CONTINUITY FOR TWO-PHASE MEDIUM}

\subsubsection{TOTAL FLOW}

According to work [17] by Kisiel et al., the total (skeleton + fluid) flow through the representative volume element (RVE) has the form

$$
\int_{S} \rho v_{i}^{S} n_{i} d S+\int_{S} \bar{\rho}\left(v_{i}^{l}-v_{i}^{S}\right) n_{i} d S+\int_{\Omega} \frac{\partial \rho}{\partial t} d \Omega=0
$$

Hence, using the Gauss-Ostrogradsky theorem, the equation of the continuity of the flow through a two-phase medium consisting of a fluid and a skeleton has the form

$$
\frac{d^{s} \rho}{\partial t}+\rho \dot{\varepsilon}=-\left[\rho v_{i}^{r}\right]_{i}
$$

where $\frac{d^{S}}{d t}$ stands for a material derivative expressed by the formula

$$
\frac{d^{\bullet}}{d t}=\frac{\partial^{\bullet}}{\partial t}+v_{i}^{s} \frac{\partial^{\bullet}}{\partial x_{i}}
$$

and $\dot{\varepsilon}$ denotes the rate of the change in skeleton dilatation, amounting to $v_{i, i}^{s}$.

\subsubsection{EQUATION OF FLUID FLOW CONTINUITY}

The mass balance of the fluid flowing through the RVE has the form

$$
\int_{S} \rho v_{i}^{r} n_{i} d S+\int_{\Omega} \frac{\partial \rho_{2}}{\partial t} d \Omega=0 .
$$

Hence, using the Gauss-Ostrogradsky theorem, the equation of fluid flow continuity becomes

$$
\frac{d^{r} \rho_{2}}{\partial t}+\rho_{2}(\dot{\theta}-\dot{\varepsilon})=0,
$$


where $\frac{d^{r}}{d t}$ is a material derivative expressed by the formula $\frac{d^{r}}{d t}=\frac{\partial}{\partial t}+\left(v_{i}^{l}-v_{i}^{s}\right) \frac{\partial}{\partial x_{i}}$ and $\dot{\theta}$ is the rate of variation of liquid dilatation.

Assuming that the solid phase is stationary $\left(v_{i}^{s}=0\right)$ and a compressible liquid flows through the pores, the equation of flow continuity is valid for only the liquid phase of the medium and comes down to

$$
\operatorname{div}\left(\rho_{2} \vec{v}^{l}\right)=\frac{\partial\left(\rho_{2}\right)}{\partial t} .
$$

This form of the equation of continuity was obtained in the preceding section (formula (10)) for the classic hydrodynamic model of the filtration flow.

\subsection{EQUATIONS OF MOMENTUM CONSERVATION FOR LIQUID PHASE}

For the medium's liquid phase the momentum conservation law comes down to

$$
\int_{S} \sigma n_{i} d S+\int_{S} b\left(v_{i}^{s}-v_{i}^{l}\right) d \Omega+\int_{\Omega} \rho_{2} X_{i} d \Omega=\int_{\Omega} \frac{\partial P_{i}^{l}}{\partial t} d \Omega,
$$

where $\sigma n_{j}$ stands for the diffused stress acting on total surface $S$ of RVE. Stress $\sigma$ amounts to

$$
\sigma=-p f
$$

where $p$ is the effective pressure in the fluid.

After taking into account the Gauss-Ostrogradsky theorem, equation (38) can be used to obtain the following local equation of the motion of the medium's liquid phase

$$
\sigma_{, i}+X_{i} \rho_{2}=b v_{i}^{r}+\rho_{12} \frac{\partial v_{i}^{s}}{\partial t}+\rho_{22} \frac{\partial v_{i}^{l}}{\partial t} .
$$

For the quasi-static flow the terms representing the inertial forces of the liquid and skeleton can be omitted and the equations of motion for the liquid phase can be written as

$$
\sigma_{, i}+X_{i} \rho_{2}=b v_{i}^{r} .
$$

The above equation leads to the following classic form of Darcy's law

$$
\vec{v}^{r}=k \operatorname{grad}\left(\frac{\sigma}{\rho_{2} f g}-x_{i} \delta_{i 3}\right) .
$$

Equation (42) leads to Darcy's equation where $k$ stands for the Darcy's coefficient of filtration

$$
\vec{v}=-k \operatorname{grad}(H)
$$

where $k=\rho_{2} f g / b$.

By performing the divergence operation on equation (41) one can write the momentum conservation equation in the form

$$
\operatorname{div}(\operatorname{grad}(\sigma))=(\dot{\theta}-\dot{\varepsilon}) / k_{w}
$$

which is known as the Darcy-Biot equation. In the latter, $\dot{\theta}$ is the rate of variation of liquid dilatation, $\dot{\varepsilon}$ - the rate of variation of skeleton dilatation, and $k_{w}$ is expressed by the formula

$$
k_{w}=\frac{k}{f \rho_{2} g}=\frac{k}{f^{2} \rho_{l} g} .
$$

\subsection{CONSTITUTIVE EQUATIONS}

Constitutive relations for the two-phase medium can be derived using the irreversible thermodynamics laws. The first law of thermodynamics can be written as

$$
\dot{L}+\dot{Q}=\frac{\partial}{\partial t}(U+K)
$$

where $\dot{L}$ denotes the power of the surface forces, the body forces and the viscous resistance of the liquid, $\dot{Q}$ - the amount of thermal energy flowing through the body, $U$ - internal energy and $K$ - kinetic energy. Having carried out procedures consistent with the principles of thermodynamics one gets for the isothermal process, on the basis of [11], [26], the following constitutive relations for the soil skeleton and the fluid flowing through the soil

$$
\begin{gathered}
\sigma_{i j}=c_{i j k l} \varepsilon_{k l}+\beta_{i j} \theta, \\
\sigma-\sigma_{a}=\beta_{i j} \varepsilon_{i j}+\gamma \theta,
\end{gathered}
$$

where $c_{i j k l}$ is a fourth-order tensor related to the deformations of the two-phase medium, $\beta_{i j}$ - the tensor of the coupling resulting from the mutual interaction of the medium's solid phase and liquid phase, $\gamma$ - a coefficient of fluid compressibility, $\sigma_{i j}$ - the tensor of the stress in the skeleton, $\sigma$-stress in the fluid, $\sigma_{a}$-atmospheric stress equal to 1 bar, $\varepsilon_{i j}-$ the tensor of skeleton deformation and $\theta$ - the dilatation of the fluid.

After simplifications (skeleton isotropy is assumed) the above constitutive equations become 


$$
c_{i j k l}=A \delta_{i j} \delta_{k l}+N\left(\delta_{i k} \delta_{j l}+\delta_{i l} \delta_{j k}\right),
$$

and assuming that second-order tensor $\beta_{i j}$ of the mutual interaction between the medium's skeleton and the fluid is expressed by

$$
\beta_{i j}=Q \delta_{i j}
$$

as well as assuming after Biot the denotation of the liquid compressibility constant one gets

$$
\gamma=R
$$

and the constitutive relations proposed by Biot in [8], [9]

$$
\begin{gathered}
\sigma_{i j}=2 N \varepsilon_{i j}+(A \varepsilon+Q \theta) \delta_{i j}, \\
\sigma-\sigma_{a}=Q \varepsilon+R \theta,
\end{gathered}
$$

where: $N, A$-Lame's elasticity constants according to Biot's denotations [8], [9], $R$ - a modulus of the volume compressibility of the fluid and $Q-$ a coefficient of the mutual interaction of the phases.

\subsection{EQUATION OF MOTION OF COMPRESSIBLE FLUID \\ THROUGH NON-DEFORMABLE SKELETON OF TWO-PHASE MEDIUM}

Assuming that the medium's skeleton is nondeformable, i.e., $\varepsilon_{i j}=0$ and so $\varepsilon=0$, physical relations (51) come down to the single relation:

$$
\sigma-\sigma_{a}=R \theta \text {. }
$$

Substituting the above physical relation into liquid motion equation (44) one gets the following relation for the non-steady flow of the fluid through the nondeformable two-phase medium

$$
\operatorname{div}(\operatorname{grad}(\sigma))=\frac{f \rho_{2} g}{k R} \frac{\partial \sigma}{\partial t} .
$$

If the flow of a compressible gas through a porous medium is considered, after the Boyle-Mariotte law is taken into account constitutive relation (52) becomes

$$
\sigma-\sigma_{a}=-\frac{\sigma_{a}}{(1+\theta)} \theta \text {. }
$$

Under the assumption of the incompressibility of the soil skeleton the equation of the flow of the gas through the soil comes down to the following equation of the filtration flow

$$
\operatorname{div}(\operatorname{grad}(\sigma))=\frac{f^{2} \rho_{l} g \sigma_{a}}{k \sigma^{2}} \frac{\partial \sigma}{\partial t} .
$$

As opposed to the equation which determines the flow of the liquid through the non-deformable porous medium, the equation of the flow of the gas is nonlinear.

\subsection{EQUATIONS OF FILTRATION OF COMPRESSIBLE FLUID THROUGH COMPRESSIBLE SKELETON OF TWO-PHASE MEDIUM}

Let us consider the filtration process in a twophase medium whose skeleton undergoes dilatational deformations, but no deviatoric deformations. In the case of the flow of a liquid through the pores of a two-phase medium, constitutive relations (52) come down to

$$
\begin{gathered}
\sigma_{m}=\frac{1}{3} \sigma_{i j}=K_{s} \varepsilon+Q \theta, \\
\sigma-\sigma_{a}=Q \varepsilon+R \theta,
\end{gathered}
$$

or converse relation

$$
\begin{gathered}
\varepsilon=\frac{R}{K_{s} R-Q^{2}} \sigma_{m}-\frac{Q}{K_{s} R-Q}\left(\sigma-\sigma_{a}\right), \\
\theta=-\frac{Q}{K_{s} R-Q^{2}} \sigma_{m}+\frac{K_{s}}{K_{s} R-Q^{2}}\left(\sigma-\sigma_{a}\right) .
\end{gathered}
$$

Assuming that in the two-phase medium the rate of variation in pore pressure is equal to, with the opposite-sign, the rate of variation of mean stress in the skeleton, one can assume that:

$$
\dot{\sigma}=-\dot{\sigma}_{m},
$$

which leads to the system of equations

$$
\begin{gathered}
-\dot{\sigma}=K_{s} \dot{\varepsilon}+Q \dot{\theta}, \\
\dot{\sigma}=Q \dot{\varepsilon}+R \dot{\theta} .
\end{gathered}
$$

Hence one gets the relation

$$
\dot{\varepsilon}=-\frac{Q+R}{Q+K_{s}} \dot{\theta},
$$

which leads to the relation

$$
\dot{\theta}=\frac{1}{R-Q \frac{Q+R}{Q+K_{s}}} \dot{\sigma} .
$$

Ultimately one gets the following equation of the unsteady flow 


$$
\operatorname{div}(\operatorname{grad}(\sigma))=\frac{\left(K_{s}-R\right) f^{2} \rho_{2} g}{\left(R K_{s}-Q\right) k} \dot{\sigma} .
$$

In the case of the flow of a gas through the twophase medium whose skeleton undergoes only dilatational deformations, the constitutive equations come down to

$$
\begin{aligned}
& \sigma_{m}=\frac{1}{3} \sigma_{i i}=K_{s} \varepsilon+Q \theta, \\
& \sigma-\sigma_{a}=Q \varepsilon-\frac{\sigma_{a}}{(1+\theta)} \theta .
\end{aligned}
$$

Slightly simplifying, one can assume the unsteady filtration equation in the form

$$
\begin{gathered}
\operatorname{div}(\operatorname{grad}(\sigma))=\frac{\left(K_{s} \sigma_{a}+\sigma^{2}\right) f^{2} \rho_{2} g}{\left(\sigma^{2} K_{s}+Q^{2} \sigma_{a}\right) k_{w}} \dot{\sigma} . \\
\text { 3. ONE-DIMENSIONAL } \\
\text { CONSOLIDATION } \\
\text { OF OEDOMETER SAMPLE } \\
\text { IN TERMS OF BIOT'S THEORY }
\end{gathered}
$$

\section{ONE-DIMENSIONAL CONSOLIDATION}

\subsection{ADOPTED MATHEMATICAL MODEL}

The one-dimensional problem was addressed by Jasiewicz in [16]. He considered the consolidation of a porous pillar filled with the Biot two-phase medium liquid, modelling in this way the behaviour of a soil sample filled with a liquid and subjected to uniaxial compression in an oedometer. In the case considered by Jasiewicz, the system of consolidation theory equations comes down to this simple form

$$
\begin{gathered}
\frac{\partial^{2} u}{\partial x^{2}}=-K_{1} \frac{\partial \sigma}{\partial x}, \text { where } K_{1}=\frac{H_{b}}{(R(M+2 N)}, \\
k_{w} \frac{\partial^{2} \sigma}{\partial x^{2}}=\frac{1}{R} \frac{\partial \sigma}{\partial t}-\frac{H_{b}}{R} \frac{\partial^{2} u}{\partial x \partial t},
\end{gathered}
$$

where $H_{b}=Q+R$. Jasiewicz solved the above system of equations assuming the following boundary conditions

- boundary conditions

$$
\begin{gathered}
\left.\sigma\right|_{x=0}=\left.0 \quad \sigma_{11}\right|_{x=0}=-\frac{p_{0}}{(1-f)} \eta(t), \\
\left.\frac{\partial \sigma}{\partial x}\right|_{x=h}=\left.0 \quad u\right|_{x=h}=0,
\end{gathered}
$$

where $\eta(t)$ denotes Heaviside functions and $p_{0}$ defines the magnitude of the load applied at instant $t=$ 0

- initial conditions for functions $u$ and $\sigma$

$$
\left.u\right|_{t=0}=\left.0 \quad \sigma\right|_{t=0}=0 .
$$

\subsection{SOLUTIONS FOR OEDOMETER SAMPLE}

By performing first the forward Laplace transformation and then, having obtained the solution in the Laplace space, the inverse Laplace transformation one gets the following solutions

$$
\begin{gathered}
\sigma(x, t)=\frac{4 p_{0} a H_{b}}{\pi} \\
\times \sum_{k=1}^{\infty} \frac{(-1)^{2 k-1}}{(2 k-1)} \cos \left[\alpha_{k}(h-x)\right] \exp \left(\frac{\alpha_{k}^{2} k_{w} t}{K_{1}}\right), \\
u(x, t)=p_{0} a R K_{1} \\
\times\left[(h-x)-\frac{8 H_{b} K_{1} h}{\pi^{2}} \sum_{k=1}^{\infty} \frac{\cos \left(\alpha_{k} x\right)}{(2 k-1)} \exp \left(\frac{\alpha_{k}^{2} k_{w} t}{K_{1}}\right)\right],
\end{gathered}
$$

where $\alpha_{k}=(2 k-1) \pi / 2 h$ and constant $a=-(M+N) /(M$ $+2 N)$.

The obtained solution has non-zero initial values, which means that the initial conditions are not satisfied. This problem was discussed at greater length in [26]. By observing the evolution of oedometer sample settlement one can determine the elasticity theory model constants and filtration coefficient $k$. The filtration coefficient determined in this way is consistent with filtration flow model (64) presented in Section 2.

\section{ONE-DIMENSIONAL CONSOLIDATION OF OEDOMETER SAMPLE IN TERMS OF TERZAGHI THEORY}

According to Lambe [18], soil consolidation is mathematically described by the one-dimensional Terzaghi consolidation model in the form

$$
\frac{\partial p}{\partial t}=c_{v} \frac{\partial^{2} p}{\partial z^{2}},
$$

where $p$ is the pressure of water in soil pores and $c_{v}$ is a Terzaghi consolidation coefficient defined by the relation 


$$
c_{v}=\frac{k M}{\rho_{2} g}
$$

where $M$ - an oedometric modulus.

In the case of consolidation in an oedometer with an outflow at both the sample's top and bottom, the solution of consolidation equation (60) has the form

$$
p=\frac{4 p_{0}}{\pi} \sum_{k=0}^{\infty} \frac{1}{2 k+1} \sin \left(\lambda_{k} x_{3}\right) \exp \left(-c_{v} \lambda_{k}^{2} t\right)
$$

where $p_{0}$ - the value of the vertical stress applied to the upper surface of the sample in the oedometer, $\lambda_{k}$ - the eigenvalue given by relation $(2 k+1) \pi / h$, and $h$ - the initial height of the sample in the oedometer. Using the constitutive relations for linear elasticity, but omitting the mutual interaction between the soil skeleton strains and the fluid strains, relation (17.3) leads to the following solution describing the settlement of the upper surface of the sample

$$
u=\frac{p_{0} h}{M}\left[1-\frac{8}{\pi^{2}} \sum_{k=0}^{\infty} \frac{1}{(2 k+1)} \exp \left(-c_{v} \lambda_{k}^{2} t\right)\right]
$$

where $u$ - stands for the displacement of the upper surface of the soil sample in the oedometer.

By measuring the displacements of the sample one can determine the value of consolidation coefficient $c_{v}$ and knowing the value of oedometric modulus $M$ and the specific density of the liquid one can calculate the filtration coefficient from formula (70). In the case of the Terzaghi consolidation model, filtration consolidation and rheological consolidation are distinguished. The filtration coefficient is determined on the basis of filtration consolidation, but the duration of filtration consolidation is based on, in our opinion, questionable assumptions. The rheological processes resulting from the rheological properties of the soil skeleton were investigated by Bartlewska et al. [5], [6].

\section{CONCLUSION}

The mathematical model of the filtration of a liquid through a porous medium, presented in Section 2 differs from the commonly used equation of nonsteady filtration. It emerges from the basic differences that:

- Proposed filtration flow equation (44) was derived (in accordance with the principles used in mechanics) not from the equation of the continuity of the liquid flowing through the soil, but from the momentum conservation law.
- The derived equation of the non-steady filtration flow depends on the adopted assumptions concerning the deformation of the two-phase medium's liquid and solid phases.

- In the case of the flow of a gas through a porous medium, the equation of non-steady filtration is nonlinear, as shown by equations (55) and (64).

- Continuity equation (10) adopted in the classic model is approximatesince it assumes that the density of the liquid is a function of time and does not depend on spatial variables $x_{i}$, where by material derivative $d \rho / d t$ is equal to local derivative $d \rho / d t$, which means that the convection term of the material derivative is neglected.

- The adopted constitutive relation defining the dependence between the rate of porosity variation and the rate of pore pressure variation should be regarded as incorrect.

- Another significant error is the adoption of the linear dependence between the rate of pore pressure variation and the rate of hydraulic head variation.

- It is evident that from the mathematical point of view the two consolidation theory solutions for the oedometer sample, i.e., Jasiewicz's solution based on the Biot theory and the solution based on the Terzaghi theory, are similar to the adopted assumptions; because of the Biot model adopted for describing the mechanical processes in a twophase medium Jasiewicz's solution is clearly closer to the non-steady filtration flow model since it takes into account the mutual interactions between the liquid phase and the solid phase in the basic constitutive relations.

- Due to the demonstrated inaccuracies the results of the numerical consolidation calculations do not agree with the results yielded by the classic filtration theory model.

There is still one more important argument for rejecting the incorrectly, in our opinion, formulated theory of the non-steady motion through the pores of a two-phase medium. From the mathematical point of view, it is difficult to add other terms to the fluid flow continuity equation when the flow of a fluid caused by the action of an electric field or by the ionic concentration gradient in the fluid is considered. In order to construct such a model one must define a function of electric current dissipation and a function of dissipation in the osmotic flow and take the functions into account in the momentum conservation equations, i.e., using the proposed methodology for defining equations of motion. It is also difficult to extend the classic mathematical model of 
filtration to non-isothermal processes. As shown by Strzelecki [23], a non-isothermal flow model is derived from the thermodynamics of irreversible processes through additional terms in the constitutive equations. By including additional temperaturedependent terms in the equations of motion one can obtain a model of thermofiltration. In the case of the classic model of filtration theory, there is no such a possibility of extending the model to more phenomena.

\section{REFERENCES}

[1] Auriault J.L., Dynamic Behaviour of a Porous Medium Saturated by a Newtonian Fluid, Int. J. Engng. Sc., 1980, Vol. 18.

[2] Auriault J.L., Strzelecki T., On the Electro-Osmotic Flow in a Saturated Porous Medium, Int. J. Engng. Sc., 1981, Vol. 19.

[3] Auriault J.L., Strzelecki T., Bauer J., He S., Porous Deformable Media Saturated by a Very Compressible Fluid, Eur. J. Mech. A/Solid, 1990, Vol. 9, 4.

[4] Bartlewska M., Defining parameters of effective rheological models of cohesive soils, doctoral dissertation, Wrocław University of Technology, Faculty of Geoengineering, Mining and Geology, Wrocław, 2009, (in Polish).

[5] BARtLewsKa M., Strzelecki T., Equations of Biot's consolidation with Kelvin-Voight rheological frame, Studia Geotechnica et Mechanica, 2009, Vol. XXXI, No. 2.

[6] Bartlewska M., Strzelecki T., One-dimensional consolidation of the porous medium with the Rheological KelvinVoight skeleton, Studia Geotechnica et Mechanica, 2008, Vol. XXX.

[7] Bensoussan A., Lions J.L., Papanicolaou G., Asymptotic Analysis for Periodic Structures, Holland Publishing Company, Amsterdam, 1978.

[8] Biot M.A., General Theory of three-dimensional Consolidation, J. Appl. Physics, 1941, Vol. 12.

[9] BIOT M.A., Theory of Propagation of Elastic Waves in a Fluid-Saturated porous Solid, I Law-Frequency Range, J.A.S.A, 1956, 28, 2.
[10] Coussy O., Revisiting the constitutive equations of unsaturated porous solids using a Lagrangian saturation concept, Int. J. Numer. Anal. Meth. Geomech., 2007, 31.

[11] Coussy O., Mechanics and Physics of Porous Solids, JohnWiley, 2010.

[12] DARCY H., Les fontaines publiques de la ville de Dijon, Paris, 1856.

[13] Detournay E., Cheng A.H.-D., Fundamentals of Poroelasticity, Comprehensive Rock Engineering: Principles, Practice and Projects, Vol. II, Analysis and Design Methods, Pergamon Press, Oxford 1993.

[14] DupuIT J., Etudes theoriques et practiquessur le movement des eaux dans les canauxdecouvert et a travers les terrains permeable, Paris, 1863.

[15] FoRCHEHEIMER P., Hydraulik, Leipzig 1914.

[16] JASIEWICZ K., Soil consolidation under a load of the upper surface there of, Archives of Civil Engineering, 1968.

[17] Kisiel I., Derski W., IzBicki R., Mróz Z., Soil and Rock Mechanics, Series: Technical Mechanics, Vol. VII, PWN, Warsaw 1982, (in Polish).

[18] Lambe T.W., Whitman R.V., Soil Mechanics, Arkady, Warsaw 1978, (in Polish).

[19] ŁYDŻBA D., Constitutive equations of gas-coal medium, Studia Geotechnica et Mechanica, 1991, 13(3-4).

[20] ŁyDżBA D., Application of the Asymptotic Homogenization Method in Soil and Rock Mechanics, Wrocław University of Technology Publishing House, Wrocaw 2002, (in Polish).

[21] ModFlow: http://water.usgs.gov/ogw/modflow/

[22] Polubarinova-Kochina P.J., Teoriya dvizheniya podzemnykh vod, Nauka, Moscow, 1977.

[23] STRZELECKI M., Quick sands effect on desert lands - example of filtration stability loss, Studia Geotechnica et Mechanica, 2013, Vol. XXXV, No. 1.

[24] Strzelecki T., Bauer J., Auriault J.L., Constitutive equation of a gas-filled two-phase medium, Transport in Porous Media, 1993, 10.

[25] Strzelecki T., Auriault J.L., Bauer J., Kostecki S., PuŁA W., Mechanics of HeterogeneousMedia, Theory of Homogenization, Lower Silesia Educational Publishers, 2008, (in Polish).

[26] Strzelecki T., Kostecki S., ŻAK, S., Modelling of Flows through Porous Media, Lower Silesia Educational Publishers, 2008, (in Polish).

[27] WiecZysty A., Engineering Hydrogeology, PWN, Warsaw 1982, (in Polish). 